University of Nebraska - Lincoln

DigitalCommons@University of Nebraska - Lincoln

3-8-1996

\title{
Thermal nucleation and cavitation in helium-3 fluids
}

\author{
Xiao Cheng Zeng \\ University of Nebraska-Lincoln, xzeng1@unl.edu \\ D.W. Oxtoby \\ James Franck Institute, University of Chicago \\ E. Cheng \\ University of California, Berkeley
}

Follow this and additional works at: https://digitalcommons.unl.edu/chemzeng

Part of the Chemistry Commons

Zeng, Xiao Cheng; Oxtoby, D.W.; and Cheng, E., "Thermal nucleation and cavitation in helium-3 fluids" (1996). Xiao Cheng Zeng Publications. 72.

https://digitalcommons.unl.edu/chemzeng/72

This Article is brought to you for free and open access by the Published Research - Department of Chemistry at DigitalCommons@University of Nebraska - Lincoln. It has been accepted for inclusion in Xiao Cheng Zeng Publications by an authorized administrator of DigitalCommons@University of Nebraska - Lincoln. 


\title{
Thermal nucleation and cavitation in helium-3 fluids
}

\author{
X. C. Zeng \\ Chemistry Department and Center for Materials Research and Analysis, University of Nebraska-Lincoln, \\ Lincoln, Nebraska 68588 \\ D. W. Oxtoby \\ The James Franck Institute, The University of Chicago, Chicago, Illinois 60637 \\ E. Cheng \\ Department of Chemistry, University of California, Berkeley, California 94720
}

(Received 7 July 1995; accepted 7 December 1995)

\begin{abstract}
We have investigated droplet nucleation and bubble cavitation in the quantum fluid helium-3 based on a nonlocal density-functional approach. A marked effect of droplet (or bubble) curvature on the rate of droplet nucleation or cavitation has been found. Without considering this curvature effect (as in the classical theory of nucleation) the droplet nucleation rate for helium-3 could be underestimated (i.e., near $1 \mathrm{~K}$ ) or overestimated (i.e., near $2.5 \mathrm{~K}$ ) by orders of magnitude, respectively; for bubble cavitation, the rate could be underestimated by more than twenty orders of magnitude (near 1 K). (C) 1996 American Institute of Physics. [S0021-9606(96)51710-4]
\end{abstract}

\section{INTRODUCTION}

Recent experiments on bubble cavitation in superfluid helium $-4^{1,2}$ have stimulated new theoretical investigations of thermal nucleation in helium fluids. ${ }^{3-5}$ Homogeneous cavitation is known to be difficult to achieve in the laboratory because a trace amount of impurity in liquids can trigger the heterogeneous cavitation. Liquid helium is an ideal system for studying homogeneous cavitation due to its unique properties at low temperature. For example, only helium can maintain the liquid state even at absolute zero temperature and ambient pressure, whereas all other materials crystallize, which renders liquid helium almost impurity-free.

Helium has two isotopes, helium-3 and helium-4: the latter is a superfluid below $2.1 \mathrm{~K}$. Recent experiments indicate that for a helium-4 superfluid, thermal cavitation may be preempted by quantized-vortex-induced cavitation. ${ }^{2}$ Therefore, if one were to study thermal fluctuation-induced homogeneous cavitation, helium- 3 would be a more suitable system since it becomes a superfluid only below $2.7 \mathrm{mK}$.

We have investigated thermal cavitation as well as droplet nucleation of helium-3 at low temperatures $(1 \mathrm{~K}-2.5 \mathrm{~K})$. Some preliminary results have been presented elsewhere; ${ }^{6}$ here we show the completed work. We note that thermal cavitation of helium-3 has been studied by Guilleumas and co-workers ${ }^{4}$ using a square-gradient density-functional approach that was developed earlier by others. ${ }^{7}$ Their functional uses five adjustable parameters to fit bulk properties of liquid helium and liquid-gas interfaces. The effect of curvature on the nucleation droplet and bubble is properly treated in that theory. They found that the nucleation barrier approaches zero near the spinodal; at high temperatures the classical nucleation theory yields results close to densityfunctional theory while at low temperatures the discrepancy between the two theories is very large.

In this work we use a nonlocal density-functional approach, originally developed by Dupont-Roc et al. ${ }^{8} \mathrm{We}$ extend their theory to study helium-3 fluid at finite tempera- tures. The advantage of the full nonlocal approach over the square-gradient one is three-fold: (i) more realistic molecular interactions, i.e., the finite-range nature of the Skyrme interaction $^{7}$ as well as the Lennard-Jones interatomic potential for inert gas elements, is incorporated; (ii) asymptotic behavior of surface profiles can be correctly described (which is power law behavior, as opposed to the exponential behavior predicted from the local density functional theory) ${ }^{9}$ and (iii) the nonlocal theory can be readily extended to study solid surface-induced heterogeneous nucleation. It is well known that local density-functional theories cannot predict correct fluid structures (i.e., density profile) for a fluid near a solid. ${ }^{10}$

\section{THEORY OF NUCLEATION}

\section{A. The classical approach}

The development of nucleation theory can be traced back to the twenties. Becker and Döring ${ }^{11}$ were the first to develop a theory for gas-liquid nucleation. That theory invokes the capillarity approximation in which the free energy of formation of a critical nucleus is calculated by treating the droplet as macroscopic with bulk and surface free energies relative to the background vapor. Under this approximation the effect of droplet curvature on the rate of nucleation is not taken into account. In that theory the free energy of formation for a critical droplet is given by

$$
\Delta \Omega_{\mathrm{CL}}^{*}=\frac{16 \pi \gamma^{3}}{3 \Delta p^{2}},
$$

where $\Delta p=p_{l}-p_{v}$ is the pressure difference between the liquid droplet and the vapor, and where the surface free energy $\gamma$ is estimated from the surface tension of an equilibrium planar liquid-vapor interface. If the liquid is assumed to be incompressible and the gas to be ideal, the pressure 
difference can be related to the supersaturation $S=p_{v} / P_{\text {coex }}$ ( $p_{\text {coex }}$ is the gas-liquid coexistence pressure at temperature $T)$ through the equation:

$$
\Delta p=\rho_{l} k_{B} T \ln S .
$$

The corresponding rate of droplet nucleation per unit volume can then be computed from the relation

$$
J_{\mathrm{CL}}=J_{0} e^{-\Delta \Omega_{\mathrm{CL}}^{*} / k_{B} T} .
$$

Here $J_{0}$ is the preexponential factor and can be calculated via $^{18}$

$$
J_{0} \approx \frac{\rho_{v}^{2}}{S \rho_{l}} \sqrt{\frac{2 \gamma}{\pi m}},
$$

where $m$ is the atomic mass and $\rho_{v}, \rho_{l}$ are gas and liquid coexistence densities at equilibrium.

\section{B. The density-functional approach}

In the density-functional approach, ${ }^{8}$ the grand canonical free energy of a helium-3 fluid is written as a functional of the density $\rho(\mathbf{r})$ :

$$
\Omega[\rho]=F_{\text {quan }}[\rho]+F_{\text {int }}[\rho]-\mu \int d \mathbf{r} \rho(\mathbf{r}),
$$

where $\mu$ is the chemical potential, $F_{\text {quan }}$ accounts for the quantum contribution:

$$
F_{\text {quan }}=\int d \mathbf{r}\left(\frac{\hbar^{2}}{2 m}|\nabla \phi|^{2}+f_{i d}\right)
$$

where the wave function $\phi(\mathbf{r})=[\rho(\mathbf{r})]^{1 / 2}$ and $f_{i d}$ is the free energy density of an ideal Fermi gas; $F_{\text {int }}$ accounts for interactions and correlations between helium-3 atoms:

$$
\begin{aligned}
F_{\text {int }}= & \frac{1}{2} \iint d \mathbf{r} d \mathbf{r} \rho(\mathbf{r}) \rho(\mathbf{r}) V_{l}(\mathbf{r})+\frac{1}{2} c \int d \mathbf{r} \rho(\mathbf{r}) \\
& \times[\bar{\rho}(\mathbf{r})]^{1+\delta},
\end{aligned}
$$

where $\delta$ is the Skyrme parameter ${ }^{7}$ and $\bar{\rho}(\mathbf{r})$ is the weighted density. For helium, it is natural to take for the interatomic potential $V_{l}$ the Lennard-Jones potential,

$$
V_{l}= \begin{cases}4 \epsilon\left(x^{-12}-x^{-6}\right) & \text { for }\left|\mathbf{r}-\mathbf{r}^{\prime}\right| \geqslant h_{4} \\ V_{l}\left(h_{4}\right)\left(\sigma x / h_{4}\right)^{4} & \text { for }\left|\mathbf{r}-\mathbf{r}^{\prime}\right| \leqslant h_{4}\end{cases}
$$

where $\epsilon / k_{B}=10.22 \mathrm{~K}, \sigma=2.556 \AA, h_{4}$ is the screening distance, and $x=\left|\mathbf{r}-\mathbf{r}^{\prime}\right| / \sigma$. Dupont-Roc et al. ${ }^{8}$ defined the weighted density over a sphere of radius $h_{4}$ centered at $\mathbf{r}$. The weighted density functional is particularly important for solid-liquid interfacial problems. ${ }^{12}$ For the gas-liquid interfacial problem (a weakly inhomogeneous system), on the other hand, replacing $\bar{\rho}(\mathbf{r})$ by $\rho(\mathbf{r})$ will give essentially the same result. ${ }^{10} h_{4}, c$, and $\delta$ are adjusted to reproduce the measured phase equilibria, ${ }^{7}$ giving the value $h_{4}=2.370 \AA$, $c=9.30292 \times 10^{6} \mathrm{~K} \AA^{3(1+\delta)}$, and $\delta=2.668$. The equilibrium density profiles can be determined by applying the variational principle to Eq. (5), that is,

$$
\delta \Omega / \delta \rho(\mathbf{r})=0
$$

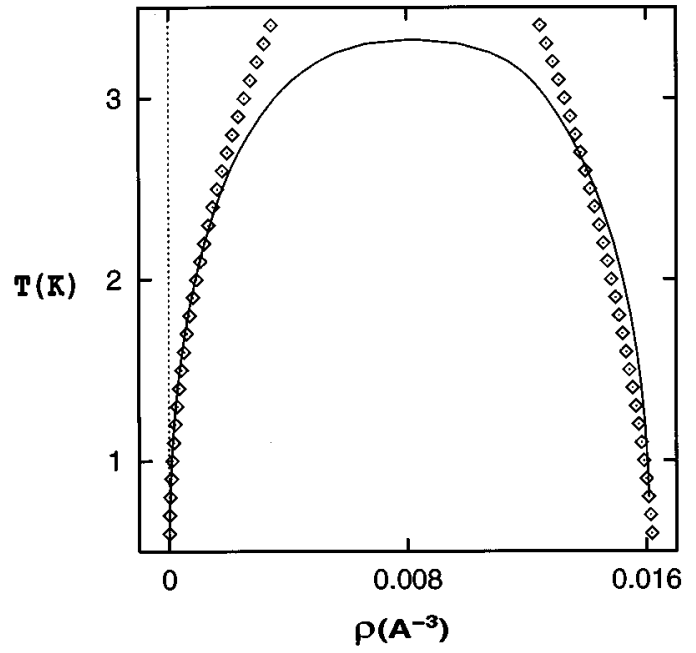

FIG. 1. Liquid-gas coexistence densities at equilibrium. The line is from experiments ${ }^{13}$ and the dots are from the present theory.

The resulting density profiles, in turn, can be substituted into Eq. (5) to compute the planar surface tensions $\gamma$.

The rate of nucleation is given by

$$
J_{\mathrm{DF}}=J_{0} e^{-\Delta \Omega_{\mathrm{DF}}^{*} / k_{B} T},
$$

where $J_{0}$ is the preexponential factor and $\Delta \Omega_{\mathrm{DF}}^{*}$ is the free energy formation of a critical droplet or bubble and is of central concern in nucleation theories because it controls the nucleation rate. $\Delta \Omega_{\mathrm{DF}}^{*}$ can also be determined from Eq. (9) since in functional space the critical droplet or bubble corresponds to a saddle point.

\section{RESULTS}

\section{A. Bulk and interfacial properties}

For a uniform density, Eqs. (6) and (7) give the freeenergy density

$$
f=f_{i d}(\rho, T)+\frac{b}{2} \rho^{2}+\frac{c}{2} \rho^{2+\delta},
$$

from which one can also derive the chemical potential

$$
\mu=\mu_{i d}+b \rho+\left(1+\frac{\delta}{2}\right) c \rho^{1+\delta},
$$

and the pressure (equation of state)

$$
p=p_{i d}+\frac{b}{2} \rho^{2}+\frac{c}{2}(1+\delta) \rho^{2+\delta} .
$$

At a given temperature $T$, the gas-liquid coexistence densities of helium-3 can be determined by solving the equations

$$
\begin{aligned}
& \mu_{l}\left(\rho_{l}, T\right)=\mu_{v}\left(\rho_{v}, T\right), \\
& p_{l}\left(\rho_{l}, T\right)=p_{v}\left(\rho_{v}, T\right) .
\end{aligned}
$$

The calculated coexistence densities are shown in Fig. 1, together with the densities from experiment. ${ }^{13}$ Like typical mean-field theory, the present theory overestimates the criti- 
cal temperature $(3.324 \mathrm{~K})$. However, below the critical temperature $(1-2.5 \mathrm{~K})$ the predicted coexistence densities agree with the measured densities very well. In this study our focus will be on helium-3 nucleation at temperatures ranging from $1 \mathrm{~K}$ to $2.5 \mathrm{~K}$.

We have also investigated planar interfacial properties, including density profiles and surface tensions. These can be obtained by solving the integro-differential equation derived from Eq. (9), which is the right hand side of Eq. (16) with the left hand being zero:

$$
i \hbar \frac{\partial \phi}{\partial t}=-\frac{\hbar^{2}}{2 m} \nabla^{2} \phi+U(\mathbf{r}, t) \phi+\left(V_{\mathrm{ext}}-\mu\right) \phi,
$$

where

$$
U(\mathbf{r}, t)=\int d \mathbf{r} \rho(\mathbf{r}, t) V_{l}\left(\left|\mathbf{r}-\mathbf{r}^{\prime}\right|\right)+\frac{c}{2}(2+\delta) \rho^{1+\delta}+\mu_{i d} .
$$

Following Dupont-Roc et al. we used the "imaginary timestep method." 8 In essence, this method is to seek solutions of Eq. (9) by adding an imaginary time derivative term on the left hand side of Eq. (9) (replacing the zero), which gives rise to Eq. (16); however, the static solutions of this newly constructed dynamic equation should be the same as those of the original Eq. (9). In fact, Eq. (16) is a time-dependent Schrödinger equation which can be formally derived by minimizing the action $\int_{t_{0}}^{t_{1}} L(t) d t$ where

$$
\begin{aligned}
L(t)= & \int d \mathbf{r}\left(\phi^{*} \frac{i \hbar}{2} \frac{\partial \phi}{\partial t}+\phi \frac{i \hbar}{2} \frac{\partial \phi^{*}}{\partial t}\right)-F[\rho] \\
& -\int d \mathbf{r}\left(V_{\mathrm{ext}}-\mu\right) \phi \phi^{*} .
\end{aligned}
$$

We summarize the method as followings: One starts with an initial guess $\phi_{0}$, chooses a time step $\Delta t$, and iterates the recursion relation $\phi_{n+1}=\exp (-H \Delta t) \phi_{n} \approx\left[1-\Delta t\left(-\hbar^{2} /\right.\right.$ $\left.\left.2 m \nabla^{2}+U\right)\right] \phi_{n}(H$ is the Hamiltonian). The ground-state eigenvalue of $H$ is the large $n$ iteration limit of $E_{n}$ which is given by $E_{n} \approx 1 / \Delta t\left(1-\left\langle\phi_{n} \mid \phi_{n+1}\right\rangle /\left\langle\phi_{n} \mid \phi_{n}\right\rangle\right)$. As shown by Dupont-Roc et al. a sensible choice for $\Delta t$ is $0.1 m(\Delta z)^{2} / 4 \hbar^{2}$ where $\Delta z$ is the distance of the mesh in $r$ space (the interface is perpendicular to the $z$-direction). The density profile of an interface can then be determined via $\rho(z)=\phi(z) \phi^{*}(z)$. The resulting density profiles at various temperatures are shown in Fig. 2. Substituting these density profiles into Eq. (5) we computed the surface tensions via the relation $\gamma=(\Omega+p V) /$ $A$, where $V$ is the volume and $A$ is the area of the interface. The calculated surface tensions are displayed in Fig. 3, together with the measured values. ${ }^{14}$ We found that the agreement between the calculation and experiments is quite reasonable.

\section{B. Droplet and bubble nucleation}

In classical nucleation theory the density profile of a nucleation droplet is assumed to be a step function with zero width. This is obviously an oversimplified picture for the interface. In the present theory this assumption is removed

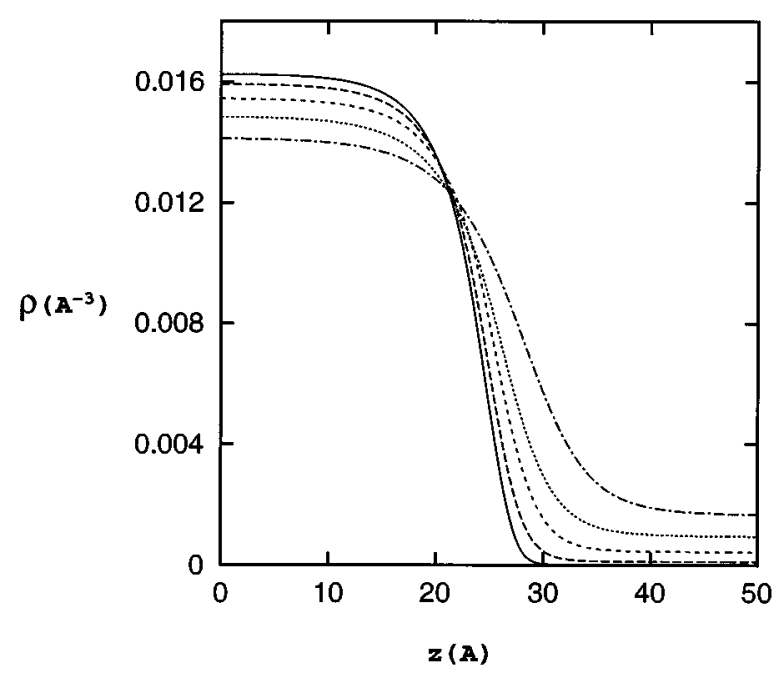

FIG. 2. The density profiles of the equilibrium planar interface at temperature $T=0.5 \mathrm{~K}$ (solid curve), $1.0 \mathrm{~K}$ (long dashed curve), $1.5 \mathrm{~K}$ (dashed curve), $2.0 \mathrm{~K}$ (dotted curve), and $2.5 \mathrm{~K}$ (dotted-dashed curve).

and the density profile of the critical droplet is determined by solving Eq. (9). The imaginary time-step method was used in conjunction with one more loop of iteration, ${ }^{15}$ due to the fact that the critical droplet is intrinsically unstable and could either grow or shrink. Although the true solution for the critical droplet should be independent of number of iterations, the second loop of iteration is required to search for the "physical" solution. Fig. 4(a) is a plot of the calculated free energy of formation $\Delta \Omega_{\mathrm{DF}}^{*}$ of the critical droplet as a function of chemical potential change $\Delta \mu=\mu-\mu_{\text {coex }}$ (characterizes the degree of supersaturation). The chemical potential change is obtained by fixing $J_{\mathrm{CL}}=1 \mathrm{~cm}^{-3} \mathrm{~s}^{-1}$. With the same $\Delta \mu$, we calculated the density profiles at various tempera-

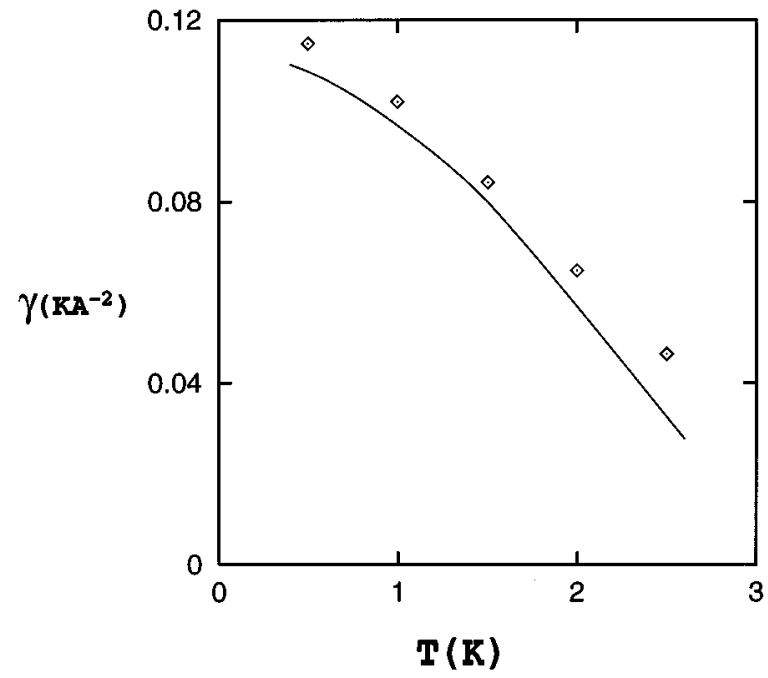

FIG. 3. The planar gas-liquid surface tension vs temperature. The dots are calculated from this theory and the line is from experimental measurement (Ref. 14). 


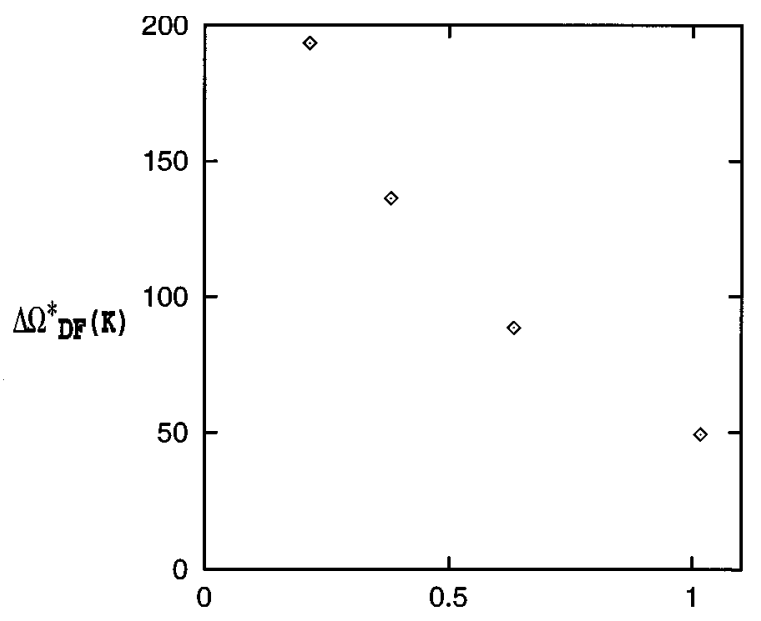

(a)
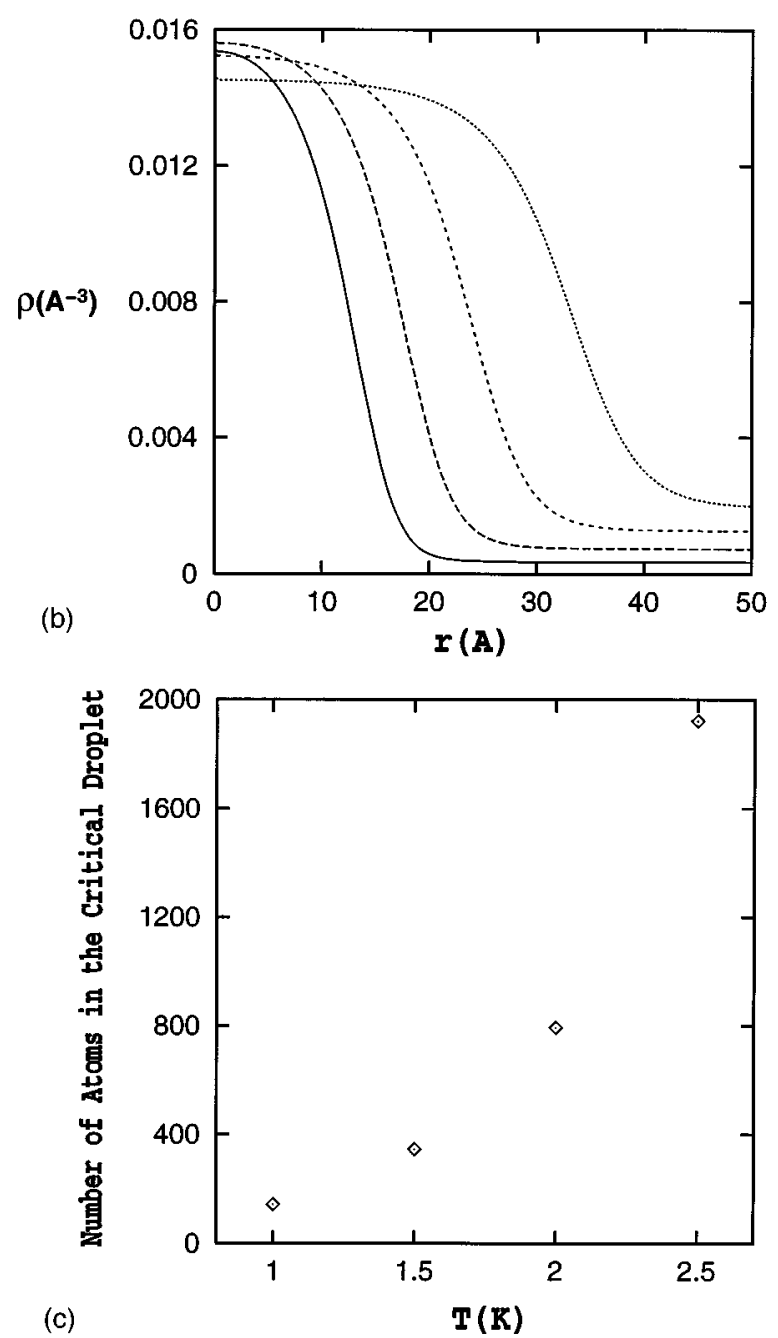

FIG. 4. (a) The free energy of formation of a critical droplet as a function of chemical potential change $\Delta \mu$ (such that $J_{\mathrm{CL}}=1 \mathrm{~cm}^{-3} \mathrm{~s}^{-1}$ ). (b) The density profile of critical droplets at temperature $T=1.0 \mathrm{~K}$ (solid curve), $1.5 \mathrm{~K}$ (long dashed curve), $2.0 \mathrm{~K}$ (dashed curve), $2.5 \mathrm{~K}$ (dotted curve). (c) The number of helium-3 atoms in a critical droplet as a function of temperature.

tures [Fig. 4(b)] and the number of atoms in a critical droplet [Fig. 4(c)]. Typically, the latter ranges from hundreds (around $1 \mathrm{~K}$ ) to thousands (around $2.5 \mathrm{~K}$ ). Next, we calcu-

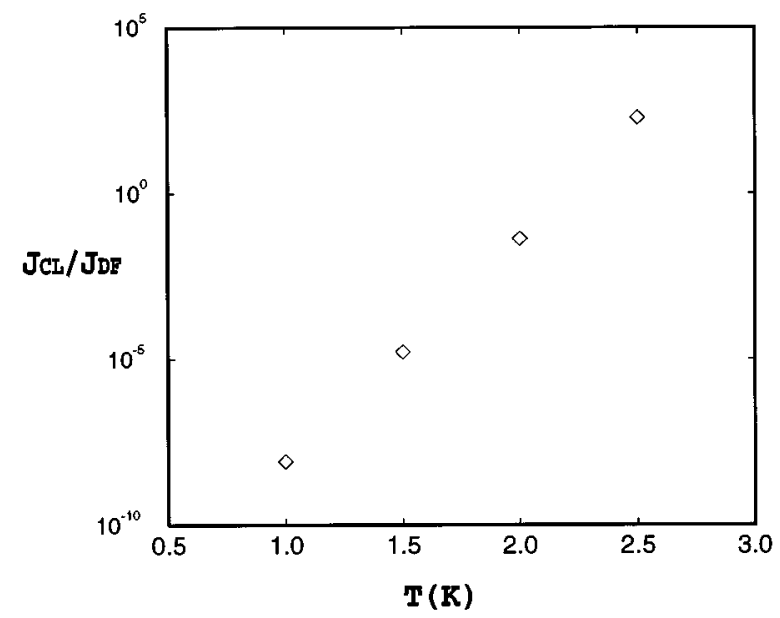

FIG. 5. Ratio of gas-to-liquid nucleation rates (classical theory to density functional theory) vs temperature.

lated $J_{\mathrm{DF}}$ as well as the temperature dependence of the ratio $J_{\mathrm{CL}} / J_{\mathrm{DF}}$ (we used the same preexponential factor $J_{0}$ in both classical and density-functional theory since it is a much weaker function of temperature $\left.{ }^{4,16}\right)$. Results are shown in Fig. 5, where $J_{\mathrm{CL}}$ is again fixed at $1 \mathrm{~cm}^{-3} \mathrm{~s}^{-1}$. We found that the classical theory overestimates the rate by two orders of magnitude at $T=2.5 \mathrm{~K}$ and underestimates the rate by eight orders of magnitude at $T=1.0 \mathrm{~K}$.

We have also investigated the bubble nucleation (cavitation) of helium-3 liquid. In this case the chemical potential change $\Delta \mu$ is negative, the density profile of a bubble has a gaslike density at its center and a liquidlike density outside. The free energy of formation against the chemical potential change is plotted in Fig. 6(a) and the density profiles of critical bubbles at various temperatures are plotted in Fig. $6(\mathrm{~b})$. Finally the temperature dependence of $J_{\mathrm{CL}} / J_{\mathrm{DF}}$ is shown in Fig. 7. Here we found considerably larger curvature effects on bubble nucleation and we note that the classical theory could underestimate the rate by more than twenty orders of magnitude at $1 \mathrm{~K}$. Indeed, this study confirms that the curvature effect cannot be neglected for 3D liquid-gas nucleation. ${ }^{1,4,15}$

The tensile strength of a liquid is related to bubble nucleation. The tensile strength $\left(P_{n}\right)$ characterizes the negative pressure a liquid can sustain before bubbles nucleate. Maris and Xiong ${ }^{1}$ proposed an empirical criterion to determine the tensile strength: the liquid is likely to break down when $J_{\exp } V_{l} \tau \sim 1$, where $J_{\exp }$ is the measured bubble nucleation rate, $V_{l}$ the liquid volume, and $\tau$ the duration of the negative pressure pulse. The product of liquid volume and time $V_{l} \tau$ is taken to be $\sim 10^{-14} \mathrm{~cm}^{3}$ s by Xiong and Maris. ${ }^{1}$ Using this criterion we have estimated the tensile strength of a helium-3 liquid at $1.5 \mathrm{~K}$ (where $J_{\mathrm{DF}} \sim 10^{14} \mathrm{~cm}^{-3} \mathrm{~s}^{-1}$ ) to be about -1.2 bar. This closes to -1.3 bar from the square-gradient density-functional theory. ${ }^{4,19}$ In contrast, the tensile strength calculated from classical theory is -1.67 bar (at $1.5 \mathrm{~K}$ ). 

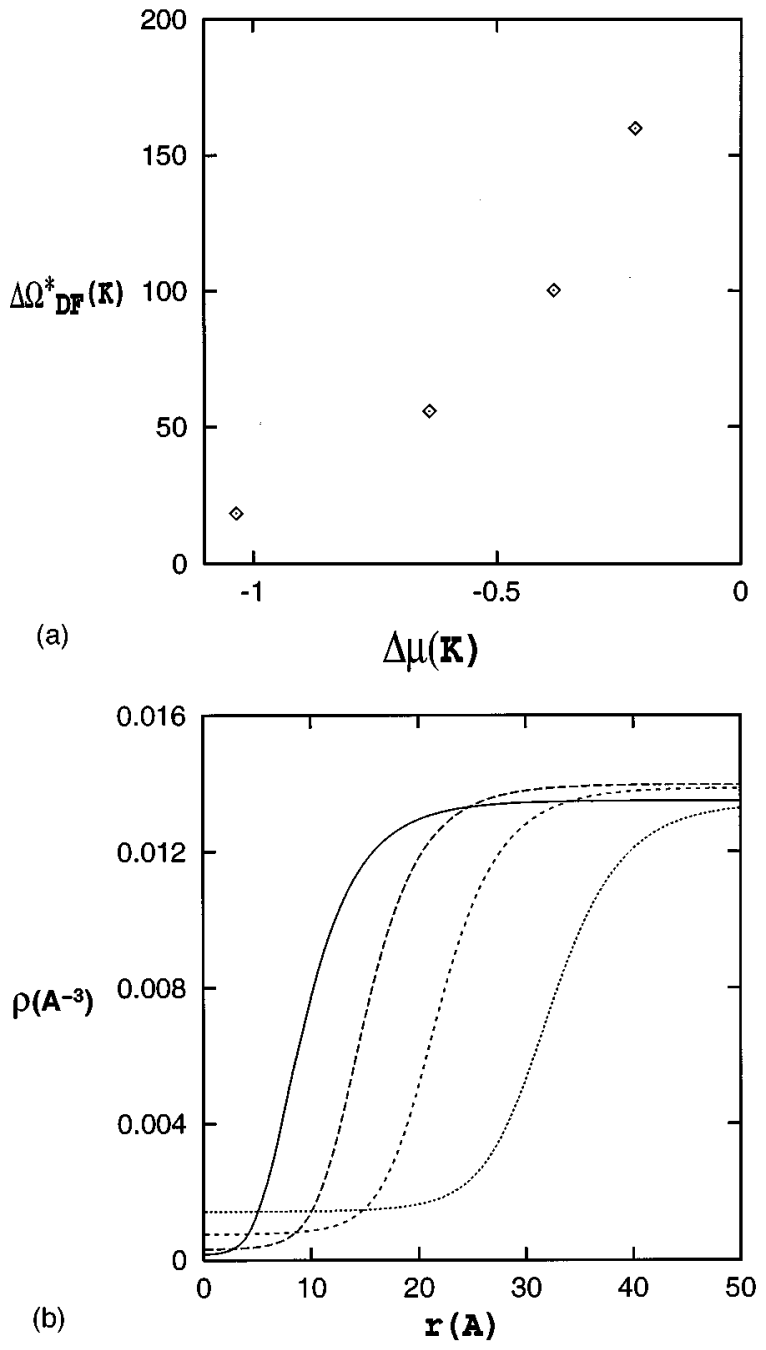

FIG. 6. (a) The free energy of formation of a critical bubble as a function of chemical potential change $\Delta \mu$ (such that $J_{\mathrm{CL}}=1 \mathrm{~cm}^{-3} \mathrm{~s}^{-1}$ ). (b) The density profile of critical bubbles at temperature $T=1.0 \mathrm{~K}$ (solid curve), $1.5 \mathrm{~K}$ (long dashed curve), $2.0 \mathrm{~K}$ (dashed curve), $2.5 \mathrm{~K}$ (dotted curve).

\section{DISCUSSION AND CONCLUSION}

We have studied gas-liquid nucleation for quantum fluid helium-3 using a nonlocal density-functional theory. This theory has the advantage that effects such as curvature dependence of the surface tension appear naturally and molecular-level detail can be incorporated if an accurate enough functional is employed. In this work we found typical nucleation droplets to be strongly curved and quite small, consisting of several tens to several hundreds of atoms [Fig. 4(c)]. Most of the atoms are at the interface [Fig. 4(b)]. Therefore, one expects that the curvature of the nucleating droplet should play an important role in nucleation and use of a planar surface tension and a sharp separation between interface and bulk (as in classical nucleation theory) are questionable.

We showed that without taking into account the curvature effect the predicted droplet nucleation rate could be off by orders of magnitude; even worse predictions result for

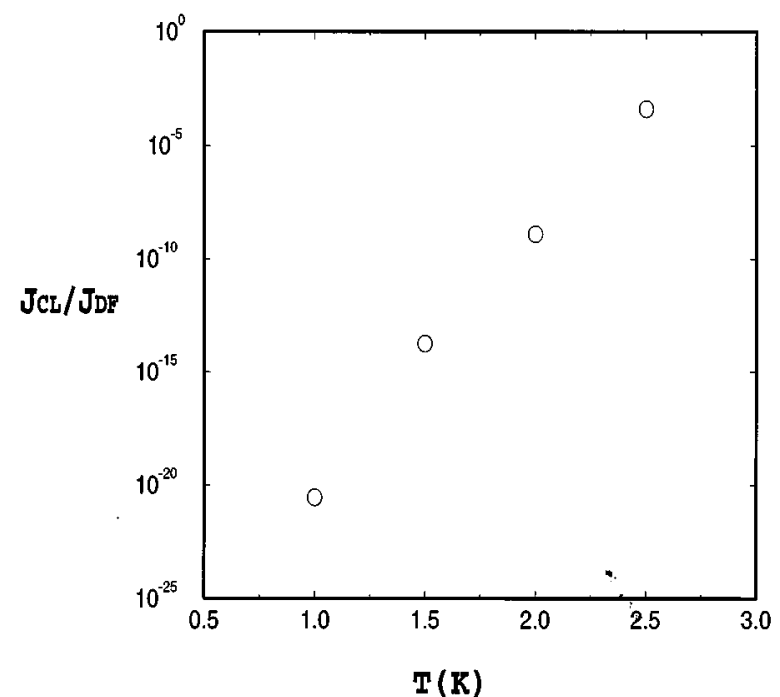

FIG. 7. Ratio of liquid-to-gas nucleation rates (classical theory to density functional theory) vs temperature.

bubble nucleation. The conclusion is the same as that from square-gradient density-functional calculations. ${ }^{1,4}$ Obviously quantitative examination of this prediction must await experiments for helium-3. Nonetheless, we can still make some qualitative comparisons with the experiments for $n$-nonane nucleation. ${ }^{17}$ We found our results are consistent with the data summarized in Ref. 17, that is, the temperature dependence of the rate ratio $J_{\mathrm{CL}} / J_{\mathrm{DF}}$ for helium-3 looks much the same as that of $J_{\mathrm{CL}} / J_{\exp }$ for $n$-nonane in Ref. 17. This is perhaps the most significant result of the present work.

\section{ACKNOWLEDGMENTS}

We are grateful to Professor J. Treiner for useful communications. This work was supported by the National Science Foundation (grants CHE-9422999, CTS-9413301, and INT-9403026) and by the Research Council of the University of Nebraska-Lincoln. Acknowledgement is also made to the donors of the Petroleum Research Fund administered by the American Chemical Society for support of this work.

${ }^{1}$ H. J. Maris and Q. Xiong, Phys. Rev. Lett. 63, 1078 (1989).

${ }^{2}$ M. S. Pettersen, C. Naud, S. Balibar, and H. J. Maris, Physica B194, 575 (1994); H. J. Maris and S. Balibar, ibid. B194, 709 (1994); M. S. Pettersen, S. Balibar, and H. J. Maris, Phys. Rev. B49, 12062 (1994); H. J. Maris, S. Balibar, and M. S. Pettersen, J. Low Temp. Phys. 93, 1069 (1993).

${ }^{3}$ Q. Xiong and H. J. Maris, J. Low Temp. Phys. 77, 347 (1989).

${ }^{4}$ D. M. Jezek, M. Guilleumas, M. Pi, M. Barranco, and J. Navarro, Phys. Rev. B48, 16582 (1993); M. Guilleumas, M. Pi, M. Barranco, J. Navarro, and M. A. Solis, ibid. B47, 9116 (1993).

${ }^{5}$ H. J. Maris, J. Low Temp. Phys. 98, 403 (1995).

${ }^{6}$ X. C. Zeng, E. Cheng, and D. W. Oxtoby, Proceedings of International Symposium on Thermodynamics in Chemical Engineering and Industry, (Chemical Industry and Engineering Society of China, Beijing, 1994), p. $245-250$.

${ }^{7}$ S. Stringari and J. Treiner, Phys. Rev. B36, 8369 (1987); F. Dalfovo and S. Stringari, J. Low Temp. Phys. 77, 307 (1989); M. Barranco, M. Pi, A. Polls, and X. Vinas, ibid. 80, 77 (1990).

${ }^{8}$ J. Dupont-Roc, M. Himbert, N. Pavloff, and J. Treiner, J. Low Temp. Phys. 81, 31 (1990). 
${ }^{9}$ See, for example, R. Evans, Adv. Phys. 28, 143 (1979).

${ }^{10} \mathrm{See}$, for example, Inhomogeneous Fluids, edited by D. Henderson (Dekker, New York, 1991).

${ }^{11}$ R. Becker and W. Döring, Mol. Phy. 24, 719 (1935); Also see J. Frenkel, Kinetic Theory of Liquids (Dover, New York, 1955).

${ }^{12}$ E. Cheng, M. W. Cole, W. F. Saam, and J. Treiner, Phys. Rev. Lett. 67, 1007 (1991).

${ }^{13}$ R. H. Sherman, Phys. Rev. Lett. 15, 141 (1965).

${ }^{14}$ M. Iino, M. Suzuki, A. J. Ikushima, and Y. Okuda, J. Low Temp. Phys. 59, 291 (1985); A. J. Ikushima, M. Iino, and M. Suzuki, Can. J. Phys. 65, 1505 (1987).
${ }^{15}$ X. C. Zeng and D. W. Oxtoby, J. Chem. Phys. 94, 4472 (1991).

${ }^{16}$ The correction of the preexponential factor only shifts absolute rates relative to the classical prediction, but cannot significantly affect the dependence of those rates on temperature or on supersaturation.

${ }^{17}$ C. Hung, M. J. Krasnopoler, and J. L. Katz, J. Chem. Phys. 90, 1856 (1989).

${ }^{18}$ D. W. Oxtoby, J. Condens. Matter 4, 7627 (1992).

${ }^{19}$ Guilleumas et al. (Ref. 4) used a different form of preexponential factor $J_{0}$ and product $V_{l} \tau\left(=2.5 \times 10^{-13} \mathrm{~cm}^{3} \mathrm{~s}\right.$ as in Ref. 1). They showed the resulting tensile strength is insensitive to the various forms of $J_{0}$. 